\title{
Non-destructive assay of nuclear materials using a self-indication method
}

\author{
Jun-ichi Hori ${ }^{1, \text { a }}$, Tadafumi Sano ${ }^{1}$, Yoshiyuki Takahashi ${ }^{1}$, Jaehong Lee ${ }^{2}$, Hironobu Unesaki ${ }^{1}$, and Ken Nakajima ${ }^{1}$ \\ 1 Kyoto University Research Reactor Institute, Asashiro-Nishi, Kumatori-cho, Sennan-gun, Osaka 590-0494, Japan \\ 2 Department of Nuclear Engineering, Kyoto University, Katsura, Nisikyo-ku, Kyoto 615-8540 Japan
}

\begin{abstract}
The integrity test applicable to TRU fuel containing MA with high radioactivity and high decay heat is important for safety. Neutron resonance transmission analysis is adapted for identification and quantification of nuclides in fuels by neutron time-of-flight measurement. In this work, a self-indication method was applied to the measurement of the transmitted neutron. The validation of the self-indication method was performed by using a pulsed neutron source and natural uranium samples at the KURRI-LINAC. The results show that the target areal density can be easily determined from the reduction of the counting rate around the resonances with and without sample. It was confirmed that the reduction ratio due to the neutron resonance absorption can be estimated to within $10 \%$. The numerical estimation showed that the areal density of fuel material can be determined in the range from $10^{-6}$ to $10^{-2}\left(\mathrm{~b}^{-1}\right)$ using multiple resonances and suitable thickness self-indicator.
\end{abstract}

\section{Introduction}

A fast reactor system with trans-uranium (TRU) fuel including minor-actinide (MA) is expected to be effective for the incineration of high-level radioactive waste [1]. To make the system with TRU fuel safer and more practical, the non-destructive quality control method for material accountancy and monitoring rise in temperature due to decay heat in a highly radioactive site is essential.

Therefore, the project entitled as "Development of Non-Destructive Methods Adopted for Integrity test of Next generation nuclear fuels (N-DeMAIN)" has been started in Japan from October 2014. In this project, we aim at the development of non-destructive assays for nuclear material from the point of view of nuclear densitometry, neutron and X-ray imaging, and the temperature measurement using Doppler broadening effect.

In this paper, we focus on neutron resonance densitometry (NRD) for material accountancy. Neutron resonance transmission analysis (NRTA) and neutron resonance capture analysis (NRCA) are useful NRD methods to identify and quantify the target nuclide [2]. They have already been applied to quantify nuclear materials [3-5]. Densitometry with a resonance analysis code such as REFIT [6] is used in general and can determine the areal density with high accuracy. However, the method accompanied by the fitting process makes it difficult to obtain the amount of nuclear material immediately. To make it practicable for the integrity test of nuclear fuel, a simple method with promptness is required. Therefore, we proposed a new concept of "selfindication method" in the previous work [7,8]. A similar method called "Self-Interrogation Neutron Resonance

a e-mail: hori@rri.kyoto-u.ac.jp
Densitometry (SINRD)" was reported as a NRD technique to directly measure the fissile content [9-11]. In the selfindication method, an indicator consisting of the target nuclide with a high purity is placed at the neutron beam downstream from a sample. The transmitted neutron can be measured indirectly by detecting the reaction products such as neutron capture gamma-rays or fission products from the indicator with the TOF method. The selfindication method makes it possible to enhance the change of transmission neutron flux in the resonance energy regions of the target nuclide. By comparing the change in counting rate with a relation between a reduction ratio of counting rate around the resonance energy and an areal density prepared in advance, it is possible to determine the areal density easily. In this method, the accuracy in the quantitative analysis depends on the predicted reduction ratio obtained by the simulation.

In this work, we carried out the experiment for application of the self-indication method to a natural uranium sample and verified the predictability of the selfindication reduction ratio using a Monte Carlo simulation.

\section{Experiment and calculation}

In order to verify the self-indication method, we performed the experiment using the 46-MeV electron linear accelerator at the Kyoto University Research Reactor Institute (KURRI-LINAC). The experimental arrangement is shown in Fig. 1. A water-cooled Ta assembly was used as a photo-neutron source [12]. The target was surrounded with a light water moderator packed in an octagonal shape aluminium case called "pac-man type". The linac was operated with a repetition rate of $250 \mathrm{~Hz}$, a pulse width of $100 \mathrm{~ns}$, a peak current of $5 \mathrm{~A}$, and an electron energy of about $30 \mathrm{MeV}$. We used a flight path in the direction of 90 degree with respect to the 


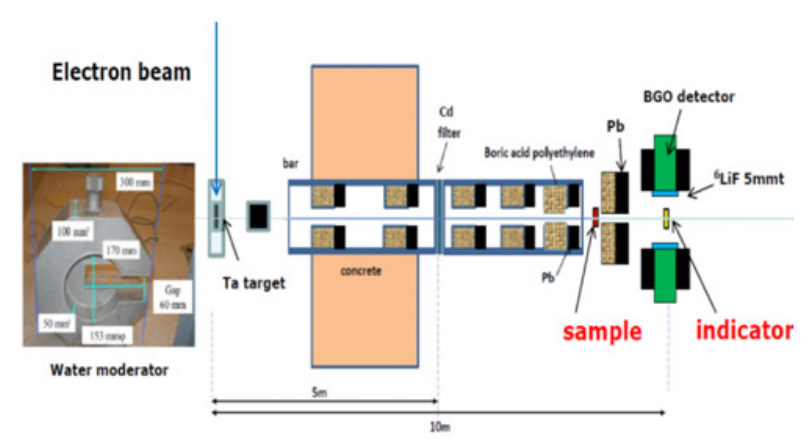

Figure 1. Experimental arrangement for the validation measurement.

linac electron beam. A Pb shadow bar was placed in front of the entrance of the flight tube to reduce the gamma flash generated by the electron burst from the Ta target. A Cd sheet of $0.5 \mathrm{~mm}$ in thickness was also inserted into the TOF neutron beam line to suppress overlap of lowenergy neutrons from the previous pulse due to the high frequency of the linac operation. Two kinds of natural uranium sheets of $40 \times 40 \mathrm{~mm}$ and $20 \times 20 \mathrm{~mm}$ were prepared as a sample and a self-indicator, respectively. In the present measurement, the areal densities of the sample and indicator were $7.36 \times 10^{-3}\left(\mathrm{~b}^{-1}\right)$. The distance between the indicator position and the neutron source was about $10 \mathrm{~m}$. Boron-mixed polyethylene and $\mathrm{Pb}$ rings and $\mathrm{B}_{4} \mathrm{C}$ shields were used to collimate the neutron beam and reduce the background. A pair of $\mathrm{Bi}_{4} \mathrm{Ge}_{3} \mathrm{O}_{12}$ (BGO) detectors, each 2 inches in diameter and 2 inches thick, was used for the measurement of capture gamma-rays from the indicator. The distance between the surface of each detector and the indicator position was $5 \mathrm{~cm}$. A ${ }^{6} \mathrm{LiF}$ tile $5 \mathrm{~mm}$ in thickness was placed at each surface of the detector to shield against scattered neutrons. Signals from the detectors were summed up and fed into a functional Multi-Channel Analyzer. Capture events detected by the detectors were taken as two-dimensional data of TOF and pulse-height $(\mathrm{PH})$, and stored in list mode. $\mathrm{A} \mathrm{BF}_{3}$ proportional counter was set at the exit of flight tube to monitor the neutron intensity during the experiment. TOF measurements of capture gamma rays from the indicator with and without the sample were carried out for 2 hours, respectively.

The TOF spectra for the capture events in the indicator with and without sample were estimated by the Monte Carlo simulation code MCNP-4C [13] with JENDL-4.0 [14]. Here, the reduction ratio $R$, which is defined as the ratio of reduction due to resonance absorption in the sample to the net resonance peak area without the sample, can be expressed with the following equation:

$$
R=\sum_{i=I_{\min }}^{I_{\max }}\left(C_{\text {out }, i}-C_{\text {in }, i}\right) / \sum_{i=I_{\min }}^{I_{\max }} C_{\text {out }, i}
$$

where $C_{i}$ is the net counting rate of the $i$-th channel in the TOF measurement and the subscripts "in" and "out" mean "with" and "without" sample. $I_{\min }$ and $I_{\max }$ correspond to the upper and lower energies for the target resonance peak, respectively. We obtained the reduction ratio $R$ for each resonance of ${ }^{238} \mathrm{U}$ by using the calculated TOF spectrum. By changing the areal density of the sample in the range from $10^{-6}$ to $10^{-1}\left(\mathrm{~b}^{-1}\right)$, the relation between

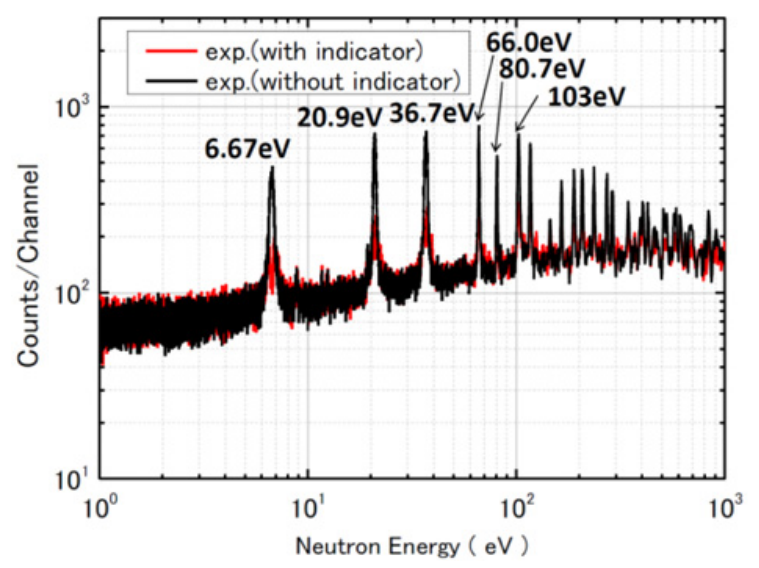

Figure 2. TOF spectra of capture event in the indicator with and without sample.

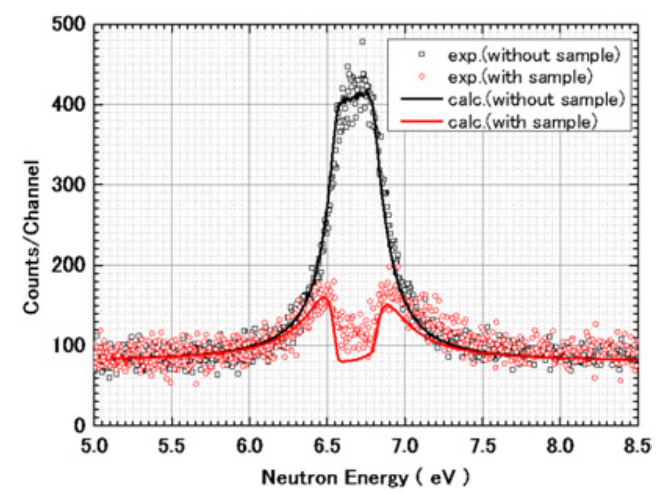

Figure 3. Comparison between experimental and calculated TOF spectra for the $6.67-\mathrm{eV}$ resonance.

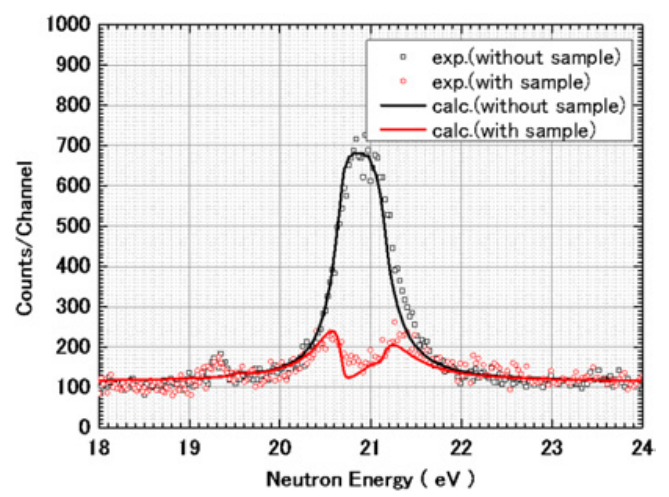

Figure 4. Comparison between experimental and calculated TOF spectra for the 20.9-eV resonance.

the reduction ratio and the areal density was calculated for each resonance.

\section{Results and discussion}

The TOF spectra with and without the natural uranium sample obtained experimentally are shown in Fig. 2. The six resonances of ${ }^{238} \mathrm{U}$ were clearly observed at 6.67 , 20.9, 36.7, 66.0, 80.7 and $103 \mathrm{eV}$. The typical comparison between experimental and calculated resonance shape are shown in Figs. 3 and 4. The calculated values were normalized so that the resonance peak area coincides with the experimental one. 
Table 1. Comparison of the experimental and calculated reduction ratio for six resonances of ${ }^{238} \mathrm{U}$.

\begin{tabular}{ccccc}
\hline Resonance $(\mathrm{eV})$ & exp. & calc. & C/E & Error(\%) \\
\hline 6.67 & $0.686 \pm 0.005$ & 0.679 & $0.99 \pm 0.01$ & 0.8 \\
20.9 & $0.683 \pm 0.008$ & 0.718 & $1.05 \pm 0.01$ & 1.2 \\
36.7 & $0.637 \pm 0.008$ & 0.702 & $1.10 \pm 0.01$ & 1.3 \\
66.0 & $0.675 \pm 0.026$ & 0.708 & $1.05 \pm 0.04$ & 3.8 \\
80.7 & $0.558 \pm 0.043$ & 0.616 & $1.10 \pm 0.08$ & 7.7 \\
103.0 & $0.689 \pm 0.031$ & 0.673 & $0.98 \pm 0.04$ & 4.5 \\
\hline
\end{tabular}

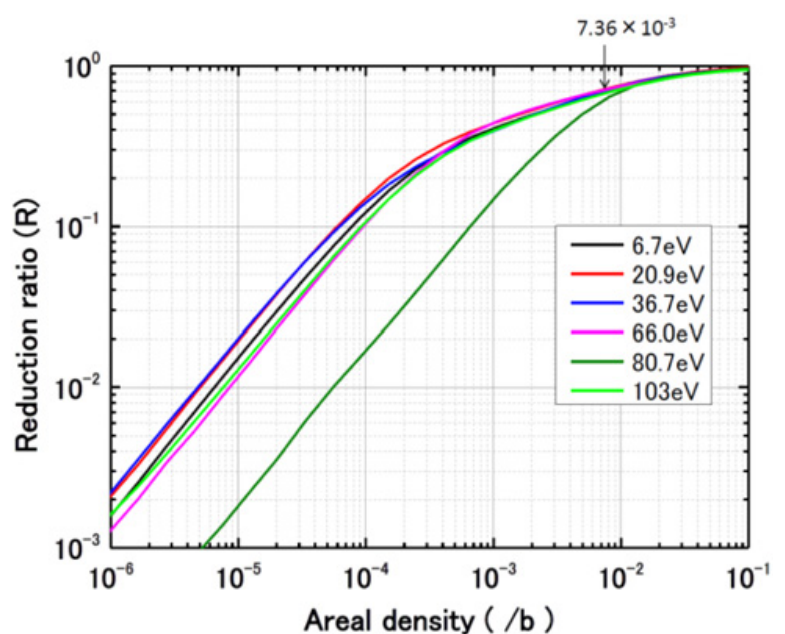

Figure 5. Relation between the reduction ratio and the areal density of the sample for each resonance of ${ }^{238} \mathrm{U}$.

In the case of low energy resonances, the shape was reproduced well by the simulation. As the resonance energy became higher, the discrepancies between the experimental and calculated resonance shapes became larger. The time resolution function for TOF measurement with the linac neutron source was not considered in the numerical calculation. Therefore, it can be considered that increasing time resolution causes the discrepancy between experimental and calculated resonance shape. It should be noted that the reduction ratio is more important than the shape in this analysis method. So the comparison between experimental and calculated reduction ratios for the six resonances is shown in Table 1. The statistical error on the resonance peak area was taken into account. The reduction ratios estimated by simple calculation reproduce the experimental ones within $10 \%$ accuracy. For the 6.67and $103-\mathrm{eV}$ resonances, the calculated results show good agreement with the experimental ones within $2 \%$. On the other hand, the reduction ratios were overestimated by the $10 \%$ for the 20.9-, 36.7-, 66.0- and 80.7-eV resonances. In order to quantify the areal density with further accuracy, it is necessary to do a more accurate calculation of the reduction ratio.

The relation between the reduction ratio and the areal density obtained from simulation with the MCNP code is shown in Fig. 5. When the areal density is not large, the reduction ratio is proportional to the areal density. However, it converges to unity and loses information about areal density as the areal density becomes larger. In the present results, the discrepancies between experiment and calculation were observed for the resonances having a large reduction ratio. Thus, the condition of the present

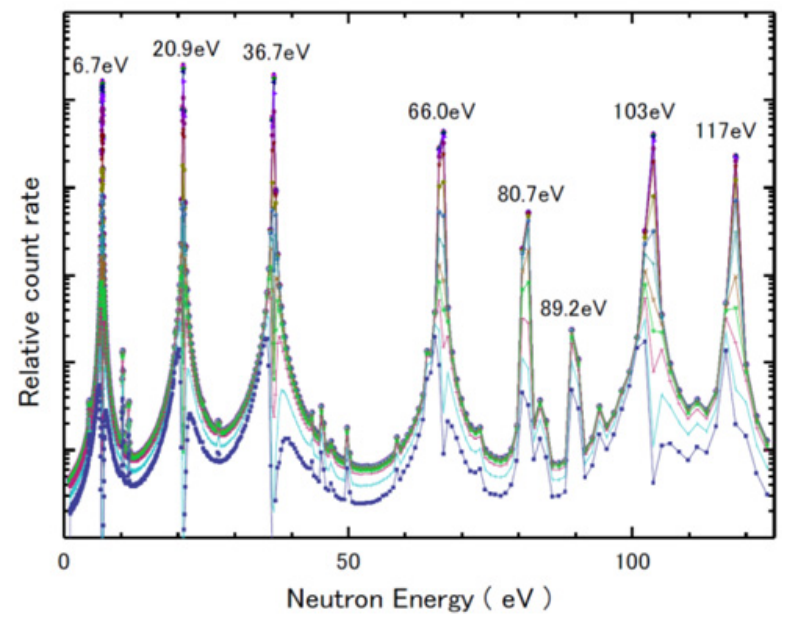

Figure 6. Calculated TOF spectra for the indicator of $1.0 \times 10^{-5}$ $\left(b^{-1}\right)$ with variable sample areal densities.

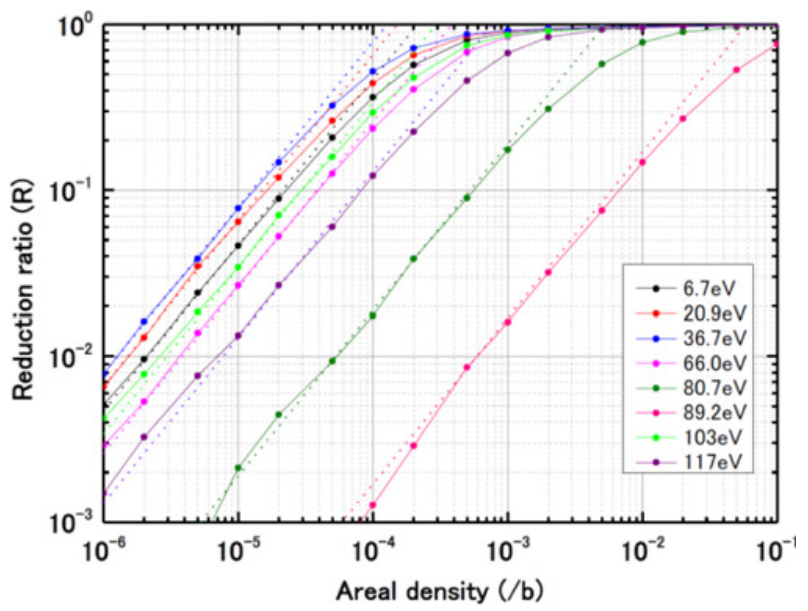

Figure 7. Relation between the reduction ratio and the areal density of the sample for each resonance of ${ }^{238} \mathrm{U}$ (areal density of indicator: $1.0 \times 10^{-5}\left(\mathrm{~b}^{-1}\right)$ ).

experiment was not suitable for accurate non-destructive assay.

We estimated the TOF spectra for the indicator of $1.0 \times 10^{-5}\left(\mathrm{~b}^{-1}\right)$ with variable sample areal densities in the range from $10^{-6}$ to $10^{-1}\left(\mathrm{~b}^{-1}\right)$ as an ideal thin indicator. The calculation results are shown in Fig. 6. The relation between the reduction ratio and the areal density for each resonance was derived as shown in Fig. 7. The linearity can be observed in the range of reduction ratio below 0.1 for every resonance. To improve the accuracy of determining the areal density, the resonance having a reduction ratio less than 0.1 should be used. On the other hand, it is desirable for the reduction ratio to be more than 0.01 from the viewpoint of statistical precision. The numerical estimation showed that the areal density of fuel material can be determined with high accuracy in the range from $10^{-6}$ and $10^{-2}\left(\mathrm{~b}^{-1}\right)$ using multiple resonances and an indicator with a suitable thickness.

\section{Summary}

We carried out a verification experiment of the selfindication method applied to a natural uranium sample at the KURRI-LINAC. The TOF spectra of the neutron 
capture events in the indicator with and without sample were experimentally and numerically obtained. In the low-energy resonance, the shape of TOF spectra can be reproduced well by a Monte Carlo simulation. Discrepancies between experimental and calculated shapes were observed as the resonance energy increases. However, it was confirmed that the reduction ratio due to resonance absorption in the sample can be estimated within $10 \%$. For the 6.67- and 103-eV resonances, the calculated results show good agreement with the experimental ones within $2 \%$.

We also calculated the relation between the reduction ratio and the areal density of the sample for the case of an ideal indicator of $1.0 \times 10^{-5}\left(\mathrm{~b}^{-1}\right)$. The numerical estimation showed that the areal density of fuel material can be determined in the range from $10^{-6}$ to $10^{-2}\left(\mathrm{~b}^{-1}\right)$ using multiple resonances and an indicator with suitable thickness.

The present works open a new possibility to determine the areal density of nuclear material by a simple area analysis with promptness. Further studies to improve the accuracy are needed.

The present study includes the result of "Development of Non-Destructive Methods Adapted for Integrity test of Next generation nuclear fuels" entrusted to the Kyoto University by the Ministry of Education, Culture, Sports, Science and Technology of Japan (MEXT).

\section{References}

[1] K. Fujimura et al., Proc. of Int. Conf. of Computational Methods and Engineering (ICCMSE), AIP Conf. Proc., 1702 (2015)

[2] P. Schillebeeckx et al., J. Instrum. 7, C03009 (2012)

[3] H.G. Priesmeyer, U. Harz, Atomkernenergie 25, 109 (1975)

[4] J.W. Behrens et al., Nucl. Technol. 67, 162 (1984)

[5] G. Noguere et al., Phys. Rev. C 74, 054602 (2006)

[6] M.C. Moxon, J.B. Brisland, AEA-InTec-0630, AEA Technology, October (1991)

[7] J. Hori et al., Nuclear Back-end and Transmutation Technology for Waste Disposal, Chap. 3, pp. 21-29, Springer (2014)

[8] T. Sano et al., Nuclear Back-end and Transmutation Technology for Waste Disposal, Chap. 4, pp. 31-37, Springer (2014)

[9] H.D. Menlove et al., Nucl. Appl. 6, 401-408 (1969)

[10] J. Hu et al., Nucl. Instrum. Meth. A 729, 247-253 (2013)

[11] R. Rossa et al., Ann. Nucl. Energy 75, 176-183 (2015)

[12] K. Kobayashi et al., Annu. Rep. Res. Reactor Inst. Kyoto Univ. 22, 142-153 (1989)

[13] J.F. Briesmeister (Ed.), LA-13709-M, Los Alamos National Laboratory (2000)

[14] K. Shibata et al., J. Nucl. Sci. Tech. 48, 1 (2011) 